PROCEEDINGS OF THE

AMERICAN MATHEMATICAL SOCIETY

Volume 138, Number 7, July 2010, Pages 2425-2430

S 0002-9939(10)10337-2

Article electronically published on March 4, 2010

\title{
INVARIANT SUBSPACES IN BERGMAN SPACE OVER THE BIDISC
}

\author{
DAVID REDETT AND JAMES TUNG
}

(Communicated by Nigel J. Kalton)

\begin{abstract}
In this paper, we investigate the doubly commuting condition restricted to invariant subspaces of the Bergman space over the bidisc. This condition was first introduced by V. Mandrekar in the setting of the Hardy space over the bidisc.
\end{abstract}

\section{INTRODUCTION}

Let $\mathbb{D}$ denote the unit disc in the complex plane. We use $H(\mathbb{D})$ to denote the collection of all functions holomorphic in $\mathbb{D}$. Let $H^{2}(\mathbb{D})$ denote the classical Hardy space. That is,

$$
H^{2}(\mathbb{D})=\left\{f \in H(\mathbb{D}): \sup _{0 \leq r<1} \int_{-\pi}^{\pi}\left|f\left(r e^{i \theta}\right)\right|^{2} d \theta<\infty\right\} .
$$

In [3, A. Beurling showed that if $\mathcal{M}$ is a (closed) subspace of $H^{2}(\mathbb{D})$ for which multiplication by the coordinate function maps $\mathcal{M}$ into itself, then $\mathcal{M}$ is of the form $\phi H^{2}(\mathbb{D})$ where $\phi$ is an inner function.

It is natural to consider the validity of Beurling's theorem in the unit bidisc. First, let us recall the definition of the classical Hardy spaces over the unit bidisc. The bidisc, $\mathbb{D}^{2}$, is the cartesian product of two copies of the unit disc. We use the notation $H\left(\mathbb{D}^{2}\right)$ to denote the collections of all functions holomorphic in $\mathbb{D}^{2}$. Holomorphic in $\mathbb{D}^{2}$ means holomorphic in each variable. The Hardy space over the bidisc is then given by

$$
H^{2}\left(\mathbb{D}^{2}\right)=\left\{f \in H\left(\mathbb{D}^{2}\right): \sup _{0 \leq r<1} \int_{-\pi}^{\pi} \int_{-\pi}^{\pi}\left|f\left(r e^{i \theta_{1}}, r e^{i \theta_{2}}\right)\right|^{2} d \theta_{1} d \theta_{2}<\infty\right\} .
$$

The question of the validity of Beurling's theorem in the bidisc becomes the following question. Is every subspace $\mathcal{M}$ of $H^{2}\left(\mathbb{D}^{2}\right)$ that is invariant under multiplication by each of the coordinate functions also generated by a single inner function? The answer to this question is no! W. Rudin showed in [6] that there are invariant subspaces $\mathcal{M}$ in $H^{2}\left(\mathbb{D}^{2}\right)$ that are not even finitely generated. Further, he showed that there are invariant subspaces of $H^{2}\left(\mathbb{D}^{2}\right)$ that contain no bounded elements,

Received by the editors September 8, 2009.

2010 Mathematics Subject Classification. Primary 47A15.

This work was done, in part, while the second author was visiting IPFW as a Scholar-inResidence.

(C)2010 American Mathematical Society Reverts to public domain 28 years from publication 
again in contrast with the one variable case, where every invariant subspace contains and is generated by a bounded function. One may then ask, what additional conditions are required so that $\mathcal{M}$ is generated by a single inner functions? In [5], V. Mandrekar answered this question by proving the following theorem.

Theorem 1 (Mandrekar). Suppose $\mathcal{M}$ is a subspace of $H^{2}\left(\mathbb{D}^{2}\right)$ invariant under $T_{z}$ and $T_{w}$. Then $T_{z}$ and $T_{w}$ doubly commute on $\mathcal{M}$ if and only if

$$
\mathcal{M}=\phi H^{2}\left(\mathbb{D}^{2}\right)
$$

where $\phi$ is an inner function.

In the above theorem we used $T_{z}$ and $T_{w}$ to denote multiplication by $z$ and $w$, respectively. We say $T_{z}$ and $T_{w}$ doubly commute on $\mathcal{M}$ when these operators commute with each other and with each other's adjoint.

Mandrekar's proof of the above theorem relied on a multivariate version of the Wold decomposition. This multivariate decomposition, due to Słociński [8], says that if $T_{1}$ and $T_{2}$ are bounded linear operators on a Hilbert space $\mathcal{H}$ for which $T_{1}$ and $T_{2}$ are isometry, doubly commute and $\bigcap_{n=0}^{\infty} T_{i}^{n}(\mathcal{H})=\{0\}, i=1,2$, then

$$
\mathcal{H}=\sum_{n=0}^{\infty} \sum_{m=0}^{\infty} \oplus T_{1}^{n} T_{2}^{m}\left(\left(\mathcal{H} \ominus T_{1}(\mathcal{H})\right) \cap\left(\mathcal{H} \ominus T_{2}(\mathcal{H})\right)\right)
$$

It is precisely this doubly commuting condition, introduced by Mandrekar, that we wish to investigate in the Bergman space over the bidisc. Before we begin this investigation, we recall some definitions and known results regarding invariant subspaces in the Bergman space.

First, we recall that the Bergman space over the unit disc is defined by

$$
A^{2}(\mathbb{D})=\left\{f \in H(\mathbb{D}): \int_{0}^{1} \int_{-\pi}^{\pi}\left|f\left(r e^{i \theta}\right)\right|^{2} r d \theta d r<\infty\right\} .
$$

In [1, A. Aleman, S. Richter and C. Sundberg showed that Beurling's theorem held in $A^{2}(\mathbb{D})$ in the following sense.

Theorem 2 (Aleman, Richter and Sundberg). Let $\mathcal{M}$ be an invariant subspace of $A^{2}(\mathbb{D})$. Then $\mathcal{M}=[\mathcal{M} \ominus z \mathcal{M}]$.

In the above theorem, $[\mathcal{M} \ominus z \mathcal{M}]$ denotes the smallest subspace of $A^{2}(\mathbb{D})$ that contains the set $\mathcal{M} \ominus z \mathcal{M}$ and is invariant under multiplication by $z$. In contrast with Beurling's theorem, where $\mathcal{M} \ominus z \mathcal{M}$ is spanned by a single inner function, C. Apostol, H. Bercovici, C. Foias and C. Pearcy in [2] showed that if $n$ is any positive integer or $\infty$, then there exists an invariant subspace $\mathcal{M}$ of $A^{2}(\mathbb{D})$ such that $\operatorname{dim}(\mathcal{M} \ominus z \mathcal{M})=n$.

Aleman, Richter and Sundberg gave a very involved, "hard" analysis proof of Theorem 2. Recently, in [7], S. Shimorin gave a "soft" analysis proof of Theorem 2, by first proving a decomposition theorem similar to the aforementioned Wold decomposition.

Our goal in this paper is to develop a multivariate version of Shimorin's theorem and use it to shed light on the doubly commuting condition in the Bergman space over the bidisc. 


\section{MAin RESUlts}

Let $\mathcal{K}$ be a subset of a Hilbert space $\mathcal{H}$ and let $T_{1}$ and $T_{2}$ be two operators on $\mathcal{H}$. We write $[\mathcal{K}]_{T_{1}}$ to denote the smallest subspace of $\mathcal{H}$ containing $\mathcal{K}$ that is invariant under $T_{1}$. Similarly, we write $[\mathcal{K}]_{T_{1}, T_{2}}$ to denote the smallest subspace of $\mathcal{H}$ containing $\mathcal{K}$ that is invariant under both $T_{1}$ and $T_{2}$.

Theorem 3. Let $T_{1}$ and $T_{2}$ be two commuting linear operators on a Hilbert space $\mathcal{H}$ with the property $\left\|T_{i} x+y\right\|^{2} \leq 2\left(\|x\|^{2}+\left\|T_{i} y\right\|^{2}\right)(x, y \in \mathcal{H}, i=1,2)$. Then,

(1) $T_{1}$ and $T_{2}$ doubly commute on $\mathcal{H}$, and

(2) $\bigcap_{n=1}^{\infty} T_{i}^{n}(\mathcal{H})=\{0\}(i=1,2)$

if and only if

(a) $\mathcal{H}=\left[\left(\mathcal{H} \ominus T_{1}(\mathcal{H})\right) \cap\left(\mathcal{H} \ominus T_{2}(\mathcal{H})\right)\right]_{T_{1}, T_{2}}$,

(b) $T_{2}$ commutes with $T_{1}^{*} T_{1}$, and

(c) $\left[\left(\mathcal{H} \ominus T_{1}(\mathcal{H})\right) \cap\left(\mathcal{H} \ominus T_{2}(\mathcal{H})\right)\right]_{T_{2}} \subseteq \mathcal{H} \ominus T_{1}(\mathcal{H})$.

To prove our theorem, we use the single operator result due to Shimorin (see [4]).

Theorem 4 (Shimorin). Let $T$ be a linear operator on a Hilbert space $\mathcal{H}$ with the properties:

(i) $\|T x+y\|^{2} \leq 2\left(\|x\|^{2}+\|T y\|^{2}\right)(x, y \in \mathcal{H})$,

(ii) $\bigcap_{n=1}^{\infty} T^{n}(\mathcal{H})=\{0\}$.

Then $\mathcal{H}=[(\mathcal{H} \ominus T(\mathcal{H}))]_{T}$.

Henceforth, we will refer to the inequality in statement (i) of Theorem 4 as the Shimorin inequality.

We now prove Theorem 3 ,

Proof. First, suppose that (1) and (2) hold. (b) follows directly from (1). To prove (a), let $E_{1}=\mathcal{H} \ominus T_{1}(\mathcal{H}), E_{2}=\mathcal{H} \ominus T_{2}(\mathcal{H})$ and $E=E_{1} \cap E_{2}$. We need to show that $\mathcal{H}=[E]_{T_{1}, T_{2}}$. Let $x \in E_{1}$. Then $T_{1}^{*} x=0$, since the ker $T_{1}^{*}=E_{1}$. Therefore, for all $y$ in $\mathcal{H}$, we have

$$
\begin{aligned}
\left\langle T_{2} x, T_{1} y\right\rangle_{\mathcal{H}} & =\left\langle T_{1}^{*} T_{2} x, y\right\rangle_{\mathcal{H}} \\
& =\left\langle T_{2} T_{1}^{*} x, y\right\rangle_{\mathcal{H}} \quad(\text { by }(1)) \\
& =0 .
\end{aligned}
$$

Therefore, $T_{2} E_{1} \perp T_{1} \mathcal{H}$, and hence $T_{2} E_{1} \subseteq E_{1}$. Similarly, we have

$$
\begin{aligned}
\left\langle T_{2}^{*} x, T_{1} y\right\rangle_{\mathcal{H}} & =\left\langle T_{1}^{*} T_{2}^{*} x, y\right\rangle_{\mathcal{H}} \\
& =\left\langle T_{2}^{*} T_{1}^{*} x, y\right\rangle_{\mathcal{H}} \quad(\text { by }(1)) \\
& =0 .
\end{aligned}
$$

Therefore, $T_{2}^{*} E_{1} \perp T_{1} \mathcal{H}$, and hence $T_{2}^{*} E_{1} \subseteq E_{1}$.

Now, let $F=E_{1} \ominus T_{2}\left(E_{1}\right)$. We now show that $F \subseteq E$. Let $x \in F$. Then $x \perp T_{2}\left(E_{1}\right)$, and hence $T_{2}^{*} x \perp E_{1}$. Also, since $x \in E_{1}$ and $E_{1}$ reduces $T_{2}$, we have $T_{2}^{*} x \in E_{1}$. Therefore, $T_{2}^{*} x=0$. Since $\operatorname{ker} T_{2}^{*}=E_{2}$, we get $x \in E_{2}$. Hence $x \in E$.

We now observe that $E_{1}=[E]_{T_{2}}$. Indeed,

$$
\begin{aligned}
E_{1} & =[F]_{T_{2}} \quad(\text { by Theorem } 4) \\
& \subseteq[E]_{T_{2}} \quad(\text { since } F \subseteq E) \\
& \subseteq E_{1} \quad\left(\text { since } E \subseteq E_{1} \text { and } E_{1} \text { is invariant under } T_{2}\right) .
\end{aligned}
$$


We point out that $F$ actually equals $E$. Also, the above sequence of containments gives us statement (c). In fact, it shows that equality actually holds in statement (c).

We finally observe that

$$
\begin{aligned}
\mathcal{H} & =\left[E_{1}\right]_{T_{1}} \quad \text { (by Theorem } 4 \text { ) } \\
& =\left[[E]_{T_{2}}\right]_{T_{1}} \quad \text { (by above) } \\
& =[E]_{T_{1}, T_{2}} .
\end{aligned}
$$

For the converse, suppose that (a), (b) and (c) hold. Let $E_{1}=\mathcal{H} \ominus T_{1}(\mathcal{H})$ and $E_{2}=\mathcal{H} \ominus T_{2}(\mathcal{H})$. If we let $E_{1}^{\prime}=\left[E_{1} \cap E_{2}\right]_{T_{2}}$, then $\mathcal{H}=\left[E_{1}^{\prime}\right]_{T_{1}}$. By supposition, $E_{1}^{\prime} \subseteq E_{1}$. Let $x$ be an element of $\mathcal{H}$. Then

$$
x=\lim _{k \rightarrow \infty} x_{k}
$$

with

$$
x_{k}=\sum_{n=0}^{N_{k}} T_{1}^{n} x_{n, k},
$$

where each $N_{k}$ is a nonnegative integer and $x_{n, k}$ is a member of $E_{1}^{\prime}$. We now have

$$
\begin{aligned}
T_{1}^{*} T_{2} x_{k} & =\sum_{n=0}^{N_{k}} T_{1}^{*} T_{2} T_{1}^{n} x_{n, k} \\
& =T_{1}^{*} T_{2} x_{0, k}+\sum_{n=1}^{N_{k}} T_{1}^{*} T_{2} T_{1}^{n} x_{n, k} \\
& \left.=\sum_{n=1}^{N_{k}} T_{1}^{*} T_{2} T_{1}^{n} x_{n, k} \quad \text { (since } T_{2} x_{0, k} \in E_{1}\right)
\end{aligned}
$$

and

$$
\begin{aligned}
T_{2} T_{1}^{*} x_{k} & =\sum_{n=0}^{N_{k}} T_{2} T_{1}^{*} T_{1}^{n} x_{n, k} \\
& =T_{2} T_{1}^{*} x_{0, k}+\sum_{n=1}^{N_{k}} T_{2} T_{1}^{*} T_{1}^{n} x_{n, k} \\
& \left.=\sum_{n=1}^{N_{k}} T_{2} T_{1}^{*} T_{1}^{n} x_{n, k} \quad \text { (since } x_{0, k} \in E_{1}\right) \\
& =\sum_{n=1}^{N_{k}} T_{1}^{*} T_{1} T_{2} T_{1}^{n-1} x_{n, k} \quad \text { (by supposition (b)) } \\
& =\sum_{n=1}^{N_{k}} T_{1}^{*} T_{2} T_{1}^{n} x_{n, k} \quad \text { (since } T_{1} \text { and } T_{2} \text { commute). }
\end{aligned}
$$

So $T_{1}^{*} T_{2} x_{k}=T_{2} T_{1}^{*} x_{k}$, and therefore $T_{1}$ and $T_{2}$ doubly commute on $\mathcal{H}$. Indeed,

$$
\begin{aligned}
\left\|T_{1}^{*} T_{2} x-T_{2} T_{1}^{*} x\right\| & \leq\left\|T_{1}^{*} T_{2} x-T_{1}^{*} T_{2} x_{k}\right\|+\left\|T_{2} T_{1}^{*} x_{k}-T_{2} T_{1}^{*} x\right\| \\
& \leq\left\|T_{1}^{*} T_{2}\right\|\left\|x-x_{k}\right\|+\left\|T_{2} T_{1}^{*}\right\|, \mid x-x_{k} \|,
\end{aligned}
$$

and letting $k$ go to infinity, we get our desired conclusion. 
By Theorem 3.6 from [7], we know that $\mathcal{H}=\left[E_{1}\right]_{T_{1}} \oplus \bigcap_{n=1}^{\infty} T_{1}^{n}(\mathcal{H})$. Since $E_{1}^{\prime} \subseteq E_{1}$ and $\mathcal{H}=\left[E_{1}^{\prime}\right]_{T_{1}}$, we may conclude that $\bigcap_{n=1}^{\infty} T_{1}^{n}(\mathcal{H})=\{0\}$. Similarly, if we define $E_{2}^{\prime}=\left[E_{1} \cap E_{2}\right]_{T_{1}}$, then $\mathcal{H}=\left[E_{2}^{\prime}\right]_{T_{2}}$. As shown above, we need only show that $E_{2}^{\prime} \subseteq E_{2}$ to conclude that $\bigcap_{n=1}^{\infty} T_{2}^{n}(\mathcal{H})=\{0\}$. To see this, let $x$ be an element of $E_{2}^{\prime}$. Then $x$ can be written as

$$
x=\lim _{k \rightarrow \infty} x_{k}
$$

with

$$
x_{k}=\sum_{n=0}^{N_{k}} T_{1}^{n} x_{n, k}
$$

where each $N_{k}$ is a nonnegative integer and $x_{n, k}$ is a member of $E_{1} \cap E_{2}$. Now, since we already showed under our assumptions that $T_{1}$ and $T_{2}$ doubly commute, we see that $T_{2}^{*} T_{1}^{n} x_{n, k}=T_{1}^{n} T_{2}^{*} x_{n, k}=0$ for every $n$ and $k$. Therefore, $T_{2}^{*} x_{k}=0$ for every $k$. Hence,

$$
\begin{aligned}
\left\|T_{2}^{*} x\right\| & =\left\|T_{2}^{*} x-T_{2}^{*} x_{k}\right\| \\
& \leq\left\|T_{2}^{*}\right\|\left\|x-x_{k}\right\|,
\end{aligned}
$$

and letting $k$ go to infinity, we get that $T_{2}^{*} x=0$. Therefore, $E_{2}^{\prime} \subseteq E_{2}$ as desired.

From this general operator theorem, we have the following theorem regarding invariant subspace of

$$
A^{2}\left(\mathbb{D}^{2}\right)=\left\{f \in H\left(\mathbb{D}^{2}\right): \int_{-1}^{1} \int_{-1}^{1} \int_{-\pi}^{\pi} \int_{-\pi}^{\pi}\left|f\left(r_{1} e^{i \theta_{1}}, r_{2} e^{i \theta_{2}}\right)\right|^{2} r_{1} r_{2} d \theta_{1} d \theta_{2} d r_{1} d r_{2}<\infty\right\} .
$$

Theorem 5. Suppose $\mathcal{M}$ is a (closed) subspace of $A^{2}\left(\mathbb{D}^{2}\right)$ invariant under $T_{z}$ and $T_{w}$. Then $T_{z}$ and $T_{w}$ doubly commute on $\mathcal{M}$ if and only if $\mathcal{M}=[(\mathcal{M} \ominus z \mathcal{M}) \cap$ $(\mathcal{M} \ominus w \mathcal{M})]_{T_{z}, T_{w}},[(\mathcal{M} \ominus z \mathcal{M}) \cap(\mathcal{M} \ominus w \mathcal{M})]_{T_{w}} \subseteq \mathcal{M} \ominus z \mathcal{M}$, and $T_{w}$ commutes with $T_{z}^{*} T_{z}$.

For any nonnegative integers $n$ and $m, \mathcal{M}=z^{n} w^{m} A^{2}\left(\mathbb{D}^{2}\right)$ satisfies the conditions of this theorem.

The only condition in Theorem 3 that needs to be verified is that $T_{z}$ and $T_{w}$ satisfy the Shimorin inequality. This is done in the following lemma.

Lemma 1. The operator $T_{z}: A^{2}\left(\mathbb{D}^{2}\right) \rightarrow A^{2}\left(\mathbb{D}^{2}\right)$ defined by

$$
\left(T_{z} f\right)(z, w)=z f(z, w)
$$

satisfies

$$
\left\|T_{z} f+g\right\|_{A^{2}\left(\mathbb{D}^{2}\right)}^{2} \leq 2\left(\|f\|_{A^{2}\left(\mathbb{D}^{2}\right)}^{2}+\left\|T_{z} g\right\|_{A^{2}\left(\mathbb{D}^{2}\right)}^{2}\right), \quad f, g \in A^{2}\left(\mathbb{D}^{2}\right) .
$$

The inequality holds when $T_{z}$ is replaced by $T_{w}$.

Before we prove this lemma, we recall a well known fact regarding the norm of a function in $A^{2}\left(\mathbb{D}^{2}\right)$. If $f$ is in $A^{2}\left(\mathbb{D}^{2}\right)$ with power series expansion $f(z, w)=$ $\sum_{m=0}^{\infty} \sum_{n=0}^{\infty} a_{m, n} z^{m} w^{n}$, then

$$
\|f\|_{A^{2}\left(\mathbb{D}^{2}\right)}^{2}=\sum_{m=0}^{\infty} \sum_{n=0}^{\infty} \frac{\left|a_{m, n}\right|^{2}}{(m+1)(n+1)} .
$$

The proof of Lemma 1 follows. 
Proof. Note that for $f(z, w)=\sum_{m=0}^{\infty} \sum_{n=0}^{\infty} a_{m, n} z^{m} w^{n}$,

$$
\left(T_{z} f\right)(z, w)=\sum_{m=0}^{\infty} \sum_{n=0}^{\infty} a_{m, n} z^{m+1} w^{n}=\sum_{m=1}^{\infty} \sum_{n=0}^{\infty} a_{m-1, n} z^{m} w^{n} .
$$

Thus

$$
\left\|T_{z} f\right\|_{A^{2}\left(\mathbb{D}^{2}\right)}^{2}=\sum_{m=1}^{\infty} \sum_{n=0}^{\infty} \frac{\left|a_{m-1, n}\right|^{2}}{(m+1)(n+1)} .
$$

Then what we need to show is that for every $f(z, w)=\sum_{m=0}^{\infty} \sum_{n=0}^{\infty} a_{m, n} z^{m} w^{n}$ and $g(z, w)=\sum_{m=0}^{\infty} \sum_{n=0}^{\infty} b_{m, n} z^{m} w^{n}$, we have

$$
\begin{gathered}
\sum_{n=0}^{\infty} \frac{\left|b_{0, n}\right|^{2}}{n+1}+\sum_{m=1}^{\infty} \sum_{n=0}^{\infty} \frac{\left|a_{m-1, n}+b_{m, n}\right|^{2}}{(m+1)(n+1)} \\
\leq 2\left(\sum_{m, n} \frac{\left|a_{m, n}\right|^{2}}{(m+1)(n+1)}+\sum_{m=1}^{\infty} \sum_{n=0}^{\infty} \frac{\left|b_{m-1, n}\right|^{2}}{(m+1)(n+1)}\right) \\
=2\left(\sum_{m=1}^{\infty} \sum_{n=0}^{\infty} \frac{\left|a_{m-1, n}\right|^{2}}{m(n+1)}+\sum_{n=0}^{\infty} \frac{\left|b_{0, n}\right|^{2}}{2(n+1)}+\sum_{m=1}^{\infty} \sum_{n=0}^{\infty} \frac{\left|b_{m, n}\right|^{2}}{(m+2)(n+1)}\right) .
\end{gathered}
$$

Thus the inequality holds if we can verify that for any integers $m$ and complex numbers $a$ and $b$,

$$
\frac{|a+b|^{2}}{m+1} \leq 2\left(\frac{|a|^{2}}{m}+\frac{|b|^{2}}{m+2}\right)
$$

this is indeed the case (see p. 277 of 4 ). That the inequality holds for $T_{w}$ is seen by exchanging the roles of $m$ and $n$.

\section{REFERENCES}

[1] A. Aleman, S. Richter and C. Sundberg, Beurling's theorem for the Bergman space, Acta. Math. 177 (1996), 275-310. MR1440934 (98a:46034)

[2] C. Apostol, H. Bercovici, C. Foias and C. Pearcy, Invariant subspaces, dilation theory and the structure of the predual of a dual algebra, J. Funct. Anal. 63 (1985), 369-404. MR808268 (87i:47004a)

[3] A. Beurling, On two problems concerning linear transformations in Hilbert space, Acta. Math. 81 (1949), 239-255. MR0027954 (10:381e)

[4] P. Duren and A. Schuster, Bergman Spaces, Math. Surveys and Monographs, 100, Amer. Math. Soc., Providence, RI, 2004. MR.2033762 (2005c:30053)

[5] V. Mandrekar, The validity of Beurling theorems in polydiscs, Proc. Amer. Math. Soc. 103 (1988), 145-148. MR938659(90c:32008)

[6] W. Rudin, Function theory in polydiscs, Benjamin, New York, 1969. MR0255841 (41:501)

[7] S. Shimorin, Wold-type decompositions and wandering subspaces for operators close to isometries, J. Reine Angew. Math. 531 (2001), 147-189. MR.1810120 (2002c:47018)

[8] M. Słociński, On the Wold-type decomposition of a pair of commuting isometries, Ann. Polon. Math. 37 (1980), 255-262. MR.587496 (83e:47031)

Department of Mathematics, Indiana University-Purdue University Fort Wayne, ForT WAYNe, INDIANA 46805

E-mail address: redettd@ipfw.edu

5701 N. Sheridan Road, Apartment 25M, Chicago, Illinois 60660

E-mail address: yanchun.tung@gmail.com 